\title{
circ-FNTA accelerates proliferation and invasion of bladder cancer
}

\author{
JIANHAI TIAN $^{1}$, JIQIANG FAN ${ }^{2}$, JIANPING XU ${ }^{1}$, TONG REN ${ }^{1}$, \\ HUAIYUAN GUO ${ }^{1}$ and LULIAN ZHOU $^{3}$ \\ ${ }^{1}$ Department of Urology Surgery, Linyi Cancer Hospital, Linyi, Shandong 276000; \\ ${ }^{2}$ Department of Emergency Surgery, The First People's Hospital of Tancheng, \\ Linyi, Shandong 276199; ${ }^{3}$ Department of Head and Neck Thoracic Surgery, \\ Linyi Cancer Hospital, Linyi, Shandong 276000, P.R. China
}

Received September 16, 2019; Accepted November 14, 2019

DOI: $10.3892 / \mathrm{ol} .2019 .11150$

\begin{abstract}
Role of circ-FNTA in the progression of bladder cancer (BCa) and its underlying mechanism were investigated. circ-FNTA level in BCa tissues and cell lines was detected. The prognostic potential of circ-FNTA was assessed by Kaplan-Meier methods and the proliferative and invasive abilities of BCa influenced by circ-FNTA were explored. Through dual-luciferase reporter gene assay, miRNA-451a, the target of circ-FNTA and the target gene of miRNA-451a, S1PR3 were determined. circ-FNTA was upregulated in BCa, especially in invasive BCa. High level of circ-FNTA indicated worse prognosis in BCa patients. Silence of circ-FNTA attenuated the proliferative and invasive abilities of T24 and UM-UC-3 cells. miRNA-451a was verified to be the target of circ-FNTA, which was downregulated in BCa cells. circ-FNTA negatively regulated the expression level of miRNA-451a. Moreover, S1PR3 was the downstream gene of miRNA-451a. Overexpression of miRNA-451a downregulated S1PR3 level in BCa cells. circ-FNTA accelerates the proliferative and invasive abilities of BCa through targeting miRNA-451a/S1PR3 axis, and indicates a poor prognosis of $\mathrm{BCa}$ patients.
\end{abstract}

\section{Introduction}

Bladder cancer (BCa) is the most common tumor in the urinary system (1). In recent years, the number of deaths caused by $\mathrm{BCa}$ has increased year by year, ranking 13th among all tumors, which poses a huge impact on human health (2). At present, therapeutic strategies, including surgery, chemotherapy and radiotherapy are applied in the treatment of

Correspondence to: Dr Lulian Zhou, Department of Head and Neck Thoracic Surgery, Linyi Cancer Hospital, 6 Lingyuan East Street, Lanshan, Linyi, Shandong 276000, P.R. China

E-mail: hp15216556900@163.com

Key words: bladder cancer, circ-FNTA, miRNA-451a, proliferation, invasion
BCa. Nevertheless, the 5-year survival of $\mathrm{BCa}$ is still low owing to the high recurrent rate and rapid progression (3). Previous studies have found that the microenvironment of tumor immunity is closely related to the progression of $\mathrm{BCa}$ (4). Some novel treatments are applied for BCa, such as the targeted drug Balversa, neoadjuvant chemotherapy and radiotherapy (5-7). It is of significance to clearly uncover the pathogenesis of $\mathrm{BCa}$, thus improving the diagnostic and therapeutic efficacies.

Development of high-throughput sequencing technology deepens gene research (8). circRNA is newly discovered and is considered to have a huge role in tumor progression (9). Previous studies have suggested that circRNA may become a potential target for tumor prediction and treatment (10). The circRNA has a cyclic structure composed of covalent bonds, characterized as high stability, high abundance, and high conservation compared with other non-coding RNAs. Functionally, circRNA is involved in rearrangement of gene information, prevention of gene degradation, and RNA folding (11). circRNAs have been reported to exert a crucial role in many types of tumors, serving as oncogenes or tumor suppressors (12-14).

A previous study demonstrated that circ-FNTA (circ_0084171) is abnormally upregulated in BCa (15). circ-FNTA locates on chr8: 42914234-42932507 with the cleavage sequence length of $582 \mathrm{bp}$. In the circbase database (http://www.circbase.org/cgi-bin/listsearch.cgi), the annotated gene of circ-FNTA is FNTA (farnesyltransferase, CAAX box, alpha, NCBI Gene 3782, transcript NM_002027) (16). Farnesyltransferase inhibitors (FTIs) are proved to inhibit the activation of multiple tumor muteins and delay tumor progression (17). It is speculated that circ-FNTA may be important in the progression of $\mathrm{BCa}$. This study mainly explored the expression pattern and biological function of circ-FNTA in $\mathrm{BCa}$, and the potential mechanism.

\section{Patients and methods}

Sample collection. BCa tissues $(\mathrm{n}=40)$ and matched normal tissues $(n=40)$ were surgically resected, immediately placed in liquid nitrogen and preserved at $-80^{\circ} \mathrm{C}$. None of enrolled $\mathrm{BCa}$ 
patients received preoperative anti-tumor therapies. Patients and their families in this study have been fully informed. This study was approved by Ethics Committee of Linyi Cancer Hospital (Linyi, China). All the patients provided written informed consent. This study was conducted in accordance with the Declaration of Helsinki.

Cell culture. Human bladder immortalized epithelium cells (SV-HUC-1) and BCa cells (5637, T24, RT4 and UM-UC-3) were provided by the American Type Culture Collection (ATCC). Cells were cultured in Roswell Park Memorial Institute 1640 (RPMI-1640) containing 10\% fetal bovine serum (FBS) (Thermo Fisher Scientific, Inc.), $100 \mathrm{U} / \mathrm{ml}$ penicillin and $100 \mu \mathrm{g} / \mathrm{ml}$ streptomycin. Cells were maintained at $37^{\circ} \mathrm{C}$, in $5 \% \mathrm{CO}_{2}$ incubator. Medium was replaced every 2-3 days.

Transfection. Transfection plasmids were provided by Sangon Biotech. Cells were pre-seeded in the 6-well plates and transfected using Lipofactamine 2000 (Invitrogen; Thermo Fisher Scientific, Inc.) at 50-70\% confluence. At 24-48 h, cells were harvested for subsequent experiments.

RNA extraction and quantitative real-time polymerase chain reaction $(q R T-P C R)$. RNA extraction from cells was performed using TRIzol reagent (Invitrogen; Thermo Fisher Scientific, Inc.). RNA was reverse transcribed into complementary deoxyribose nucleic acid (cDNA) using Primescript RT Reagent (TaKaRa). The obtained cDNA was subjected to qRT-PCR using SYBR ${ }^{\circledR}$ Premix Ex Taq ${ }^{\mathrm{TM}}$ (TaKaRa). Glyceraldehyde 3-phosphate dehydrogenase (GAPDH) and U6 were used as internal references. Each sample was performed in triplicate, and relative level was calculated by $2^{-\Delta \Delta C t}$. Primer sequences are listed in Table I.

Cell Counting Kit (CCK-8). Cells were seeded in the 96-well plate with $5 \times 10^{3}$ cells per well. At the appointed time points, absorbance value at $450 \mathrm{~nm}$ of each sample was recorded using the CCK-8 kit (Dojindo Laboratories) for depicting the viability curve.

5-Ethynyl-2'-deoxyuridine (EdU) proliferation assay. Cells were inoculated into 96 -well plates with $1 \times 10^{5}$ cells per well, and labeled with $100 \mu \mathrm{l}$ of EdU reagent $(50 \mu \mathrm{M})$ per well for $2 \mathrm{~h}$. After washing with phosphate buffered saline (PBS), the cells were fixed in $50 \mu \mathrm{l}$ of fixation buffer, decolored with $2 \mathrm{mg} / \mathrm{ml}$ glycine and permeated with $100 \mu \mathrm{l}$ of penetrant. After washing with PBS once, cells were stained with AdoLo and 4',6-diamidino-2-phenylindole DAPI) in the dark for $30 \mathrm{~min}$. EdU-positive ratio was determined under a fluorescent microscope.

Transwell invasion assay. Cell density was adjusted to $3 \times 10^{4}$ cells $/ \mathrm{ml}$. Suspension $(100 \mu \mathrm{l})$ was applied to the upper Transwell chamber (Corning Inc.). Into the lower chamber, $600 \mu \mathrm{l}$ of medium containing 20\% FBS was applied. After $24 \mathrm{~h}$ of incubation, cells migrated to the lower chamber were fixed in methanol for $15 \mathrm{~min}$, stained with crystal violet for $20 \mathrm{~min}$ and counted using a microscope. The number of migratory cells was counted in 5 randomly selected fields per sample (x200).
Target gene prediction. Target genes of circ-FNTA and miRNA-451a were predicted on Starbase (http://starbase.sysu. edu.cn/) (18) and TargetScan (http://www.Targetscan.org) (19). Predicted miRNAs on both websites were depicted by Venn diagram. The network of target genes of miRNA-451a was depicted using Cytoscape software v.3.5.1.

Dual-luciferase reporter gene assay. Based on the predicted binding sites, we constructed pmirGLO-circ-FNTA-mut, pmirGLO-circ-FNTA-wt, pmirGLO-S1PR3-mut and pmirGLO-S1PR3-wt. Cells were co-transfected with miRNA-451a mimics/NC and wild-type/mutant-type vectors using Lipofectamine 2000. After 48 h, co-transfected cells were collected for determining luciferase activity using a dual-luciferase reporter assay system (Promega Cooperation).

Statistical analysis. GraphPad Prism 6 (La Jolla) was used for data analyses. Data were expressed as mean \pm standard deviation. Intergroup differences were analyzed by the t-test. Kaplan-Meier method was introduced for survival analysis. Two-tailed $\mathrm{P}<0.05$ was considered as statistically significant.

\section{Results}

circ-FNTA is upregulated in BCa. QRT-PCR showed higher abundance of circ-FNTA in BCa tissues relative to normal ones (Fig. 1A). Similarly, its level was higher in BCa cells than that of bladder epithelial cells (Fig. 1B). According to the invasion status of the enrolled BCa patients, they were classified into non-invasive group and invasive group. circ-FNTA was upregulated in the invasive group compared with that of the non-invasive group (Fig. 1C). Through analyzing the follow-up data of BCa patients, it is found that high level of circ-FNTA predicted worse prognosis of BCa (Fig. 1D). It is suggested that circ-FNTA may exert a carcinogenic role in the progression of $\mathrm{BCa}$.

Knockdown of circ-FNTA suppresses proliferative and invasive abilities of $B C a$. T24 and UM-UC-3 cell lines were selected for the following in vitro experiments. We constructed two circ-FNTA siRNAs (si-circ-FNTA \#1 and si-circ-FNTA \#2). Transfection of si-circ-FNTA \#1 or si-circ-FNTA \#2 markedly downregulated circ-FNTA level in BCa cells (Fig. 2A). CCK-8 assay showed reduced viability in T24 and UM-UC-3 cells transfected with si-circ-FNTA \#1 or si-circ-FNTA \#2 (Fig. 2B, 2C). Knockdown of circ-FNTA markedly decreased the ratio of EdU-positive cells, suggesting inhibited proliferative ability of BCa cells (Fig. 2D). Transwell assay showed that knockdown of circ-FNTA in T24 and UM-UC-3 cells markedly decreased the ratio of invasive cells, indicating an attenuated invasive ability (Fig. 2E). Hence, silence of circ-FNTA was proved to attenuate proliferative and invasive abilities of BCa cells.

circ-FNTA targets miRNA-451a. According to the prediction on Starbase and TargetScan, a total of 29 overlapped target miRNAs of circ-FNTA were discovered (Fig. 3A). miRNA-451a is previously reported to be downregulated in $\mathrm{BCa}(20)$. It is predicted to be the downstream target of circ-FNTA among the 29 overlapped ones. Hence, we focused on the potential role of miRNA-451a in the progression of $\mathrm{BCa}$. Through bioinformatics analysis, 
Table I. Sequences of transfection primers.

\begin{tabular}{|c|c|}
\hline Genes & Primer sequence \\
\hline \multicolumn{2}{|l|}{ miRNA cDNA } \\
\hline miRNA-451a & Primer 5'-AAAAAAACCGTTACCATTACTGAGTT-3' \\
\hline U6 & Primer 5'-GCAAATTCGTGAAGCGTTCCATA-3' \\
\hline \multicolumn{2}{|l|}{ qRT-PCR primer } \\
\hline \multirow[t]{2}{*}{ circ-FNTA } & Forward 5'-GCCCAAAAACTATCAAGTTTGGCAT-3' \\
\hline & Reverse 5'-ATAACCCATTGTCGATGCTGCC-3' \\
\hline \multirow[t]{2}{*}{ S1PR3 } & Forward 5'-TCTCCGAAGGTCAAGGAAGA-3' \\
\hline & Reverse 5'-TCAGTTGCAGAAGATCCCATTC-3' \\
\hline \multirow[t]{2}{*}{ GAPDH } & Forward 5'-TCCTCTGACTTCAACAGCGACAC-3' \\
\hline & Reverse 5'-GAGCAACACAGATGAACCGC-3' \\
\hline \multirow[t]{2}{*}{ miRNA-451a } & Forward 5'-GGCCCTCGAGCTTTTGACCACCCCTTAACC-3' \\
\hline & Reverse 5'-CCCGGGGCGGCCGCACAATGAATTATAATACAAT-3' \\
\hline \multirow[t]{2}{*}{ U6 } & Forward 5'-AGAAGGCTGGGGCTCATTTG-3' \\
\hline & Reverse 5'-AGGGGCCATCCACAGTCTTC-3' \\
\hline
\end{tabular}

A

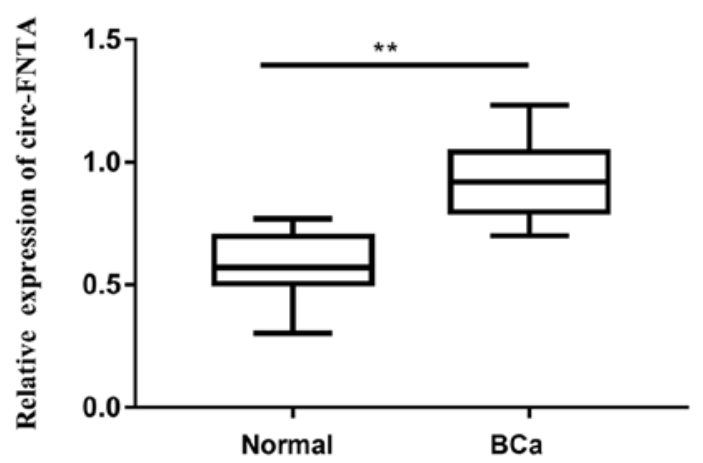

C

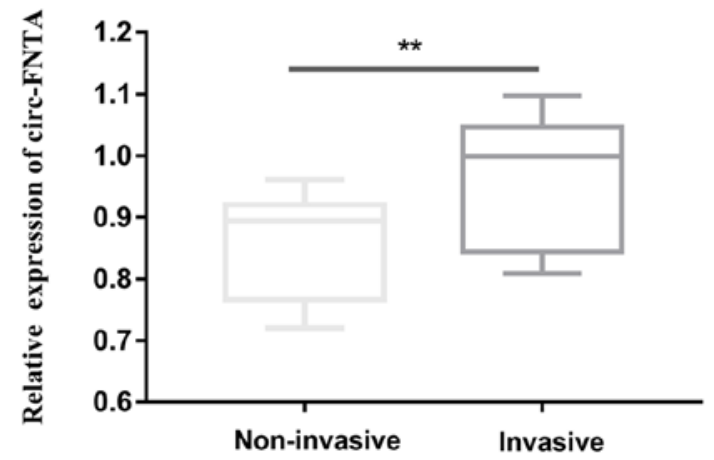

B

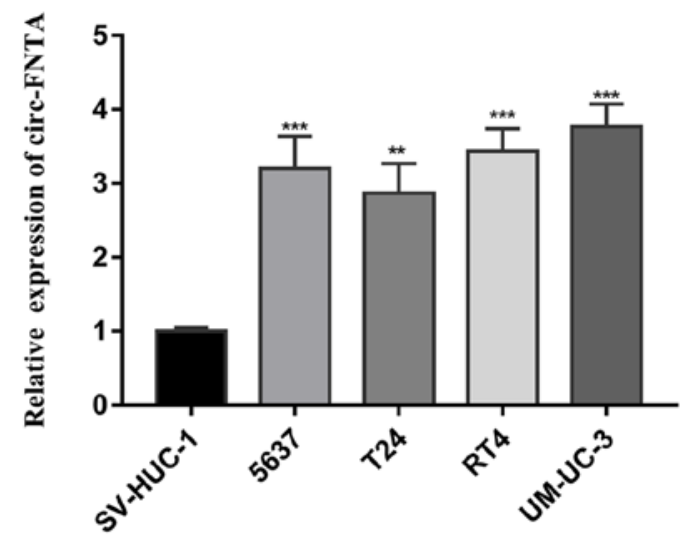

D

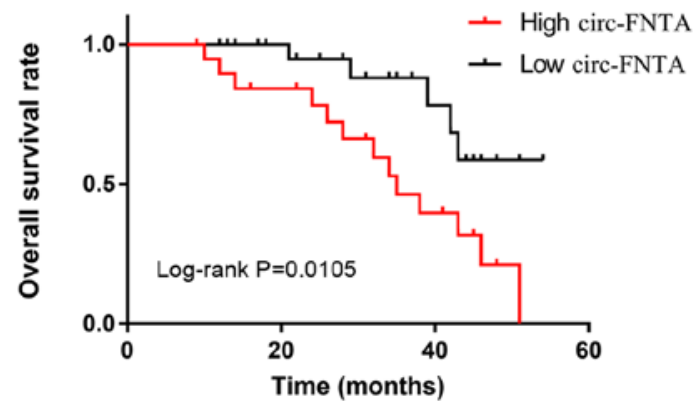

Figure 1. circ-FNTA was upregulated in BCa. (A) Relative level of circ-FNTA in BCa tissues and normal tissues (compared with normal, ${ }^{* *} \mathrm{P}<0.01$ ). (B) Relative level of circ-FNTA in human bladder immortalized epithelial cells (SV-HUC-1) and BCa cells (5637, T24, RT4 and UM-UC-3) (compared with SV-HUC-1, ${ }^{* *} \mathrm{P}<0.01,{ }^{* * * *} \mathrm{P}<0.001$ ). (C) Relative level of circ FNTA in non invasive BCa tissues and invasive BCa tissues (compared with non-invasive, $\left.{ }^{* * *} \mathrm{P}<0.01\right)$. (D) Kaplan-Meier curves of the overall survival in BCa patients with high level or low level of circ-FNTA. BCa, bladder cancer.

potential binding sites between circ-FNTA and miRNA-451a were identified (Fig. 3B). A remarkable decline in luciferase activity was observed after co-transfection of miRNA-451a mimics and pmirGLO-circ-FNTA-wt, confirming the binding relationship between circ-FNTA and miRNA-451a (Fig. 3C). Expression level of miRNA-451a was markedly upregulated 
A

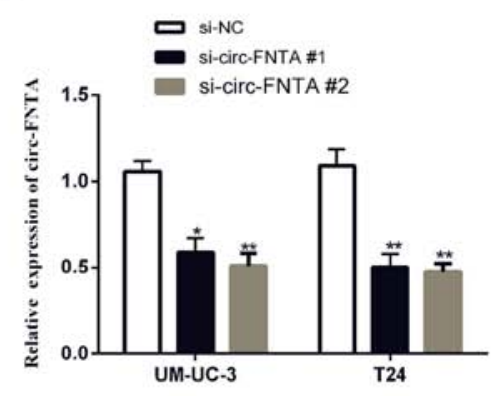

D
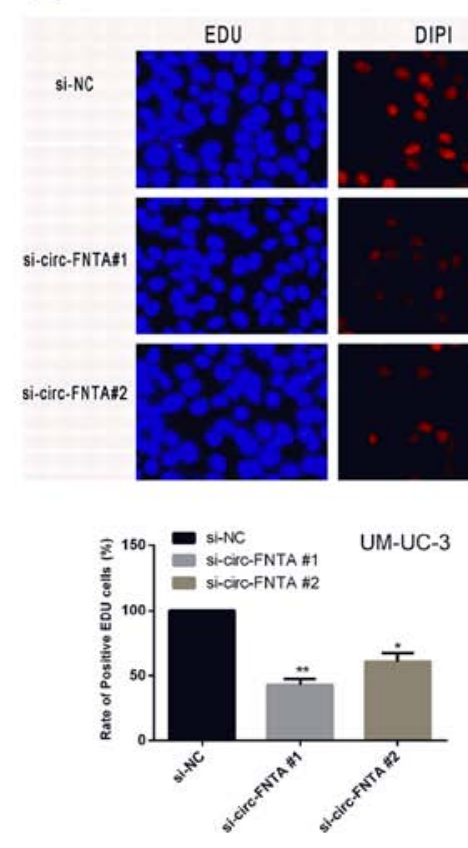

B

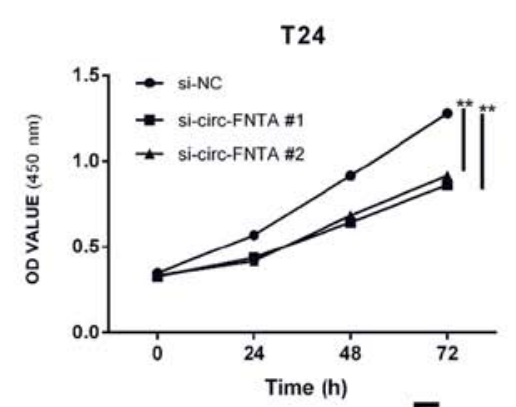

C

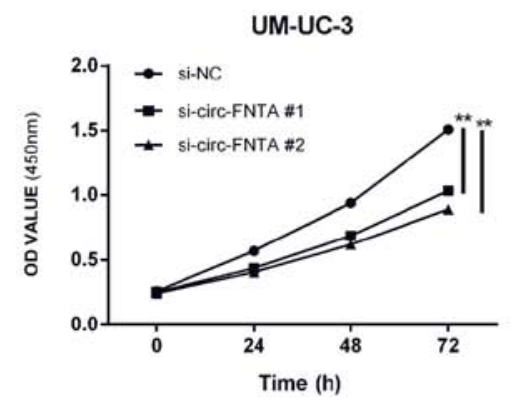

E
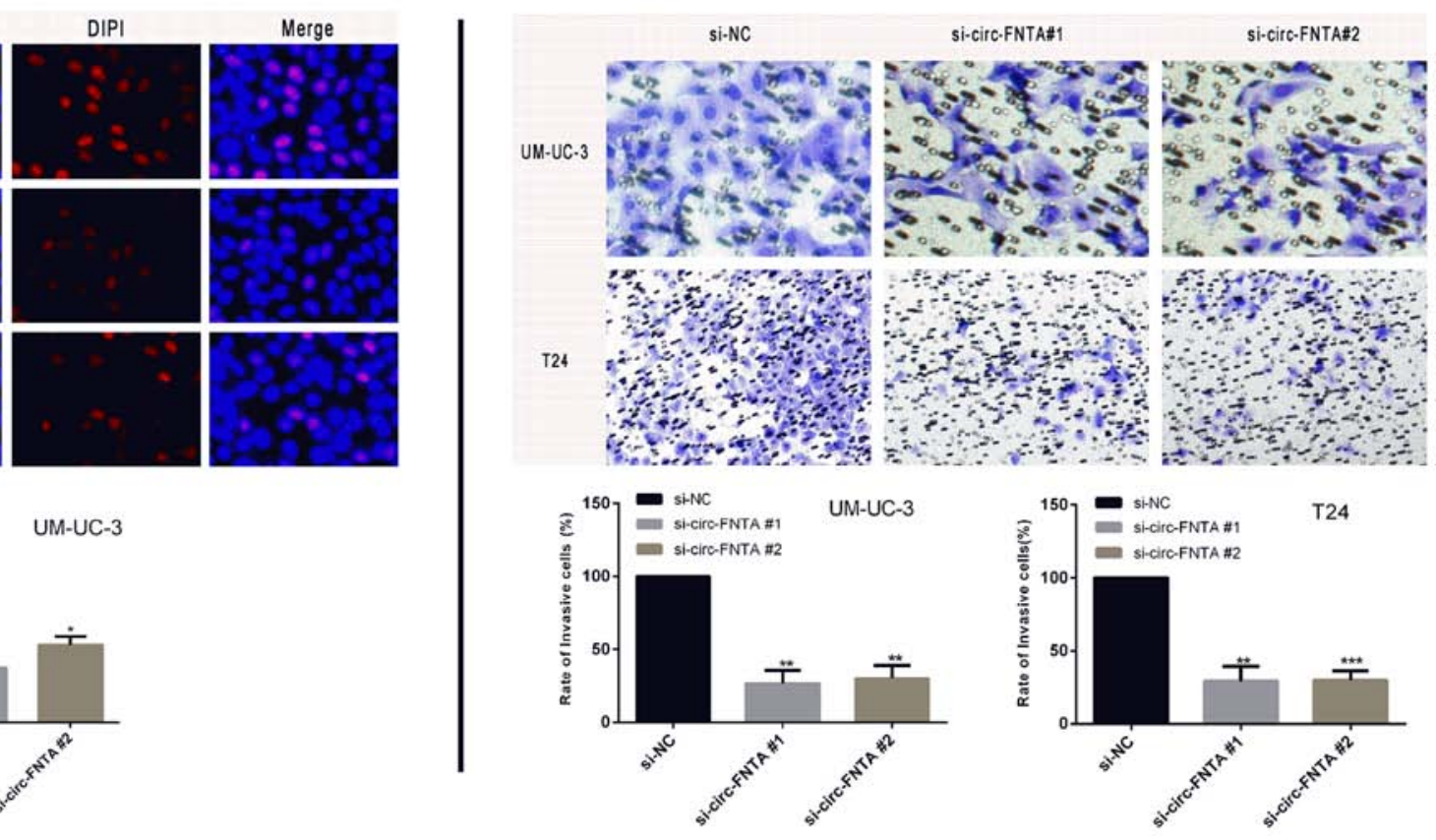

si-circ. FNTA\#1

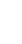

Figure 2. Knockdown of circ-FNTA suppresses proliferative and invasive abilities of BCa. (A) Transfection efficacy of si-circ-FNTA \#1 and si-circ-FNTA \#2 in UM-UC-3 and T24 cells. (B) Viability in T24 cells transfected with si-NC, si-circ-FNTA \#1 or si-circ-FNTA \#2. (C) Viability in UM-UC-3 cells transfected with si-NC, si-circ-FNTA \#1 or si-circ-FNTA \#2. (D) EdU assay of the ratio of EdU-positive cells in UM-UC-3 cells transfected with si-NC, si-circ-FNTA \#1 or si-circ-FNTA \#2. (E) Transwell assay of the ratio of invasive cells in UM-UC-3 and T24 cells transfected with si-NC, si-circ-FNTA \#1 or si-circ-FNTA \#2. $\mathrm{BCa}$, bladder cancer. Compared with si-NC, ${ }^{* *} \mathrm{P}<0.05,{ }^{* *} \mathrm{P}<0.01,{ }^{* * *} \mathrm{P}<0.001$.

by transfection of si-circ-FNTA \#1 in T24 and UM-UC-3 cells (Fig. 3D). It was found that miRNA-451a was downregulated in BCa cells relative to bladder epithelial cells (Fig. 3E). The above data demonstrated that circ-FNTA targeted miRNA-451a and negatively regulated its level in $\mathrm{BCa}$.

miRNA-451a regulates its target gene S1PR3. Through analyzing the database, S1PR3 was predicted to be the target gene of miRNA-451a (Fig. 4A). A relevant study reported the involvement of S1PR3 in the progression of $\mathrm{BCa}(21)$. Potential binding sites between miRNA-451a and S1PR3 were identified (Fig. 4B). Subsequently, dual-luciferase reporter gene assay verified the binding relationship between miRNA-451a and S1PR3 (Fig. 4C). Transfection of miRNA-451a mimics remarkably downregulated S1PR3 level in T24 and UM-UC-3 cells (Fig. 4D). In addition, S1PR3 was downregulated in $\mathrm{BCa}$ tissues compared with those of controls (Fig. 4E). As a result, S1PR3 was demonstrated to be the target gene of miRNA-451a. It is suggested that circ-FNTA/miRNA-451a/S1PR3 axis exerted carcinogenic role in $\mathrm{BCa}$.

\section{Discussion}

The role of circRNAs in urinary tumors has been well studied (22). Plenty of circRNAs have been discovered participating in the progression of $\mathrm{BCa}$. For example, circRNA-cTFRC absorbs miRNA-107 to regulate target gene expression, and thereafter aggravates the progression of $\mathrm{BCa}$ (23). CircGprc5a is upregulated in BCa. It induces the upregulation of Gprc5a through a polypeptide, and further stimulates the progression of $\mathrm{BCa}$ (24). circRNA-PRMT5 accelerates EMT of BCa through sponging miRNA-30c (25). This study mainly explored the role of circ-FNTA, a newly discovered circRNA, in regulating the progression of $\mathrm{BCa}$.

The reference gene for circ-FNTA is the FNTA gene located on chromosome 8. FNTA is considered to be a key gene for tumor progression through activating the Ras-MAPK pathway. FTI alleviates tumor progression through blocking the activation of Ras-MAPK pathway (26). Abnormal copy numbers of FNTA are believed to cause pathological changes of breast cancer, which are key targets for developing drugs (27). 
A

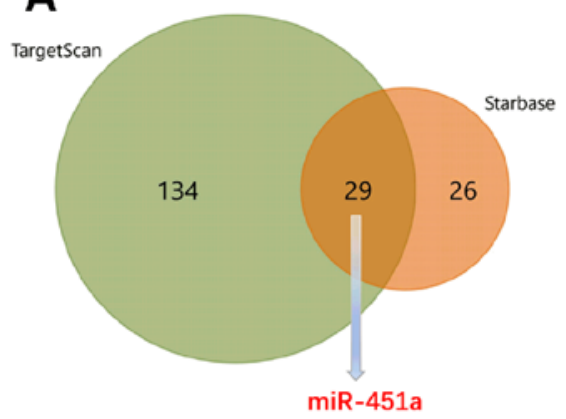

B

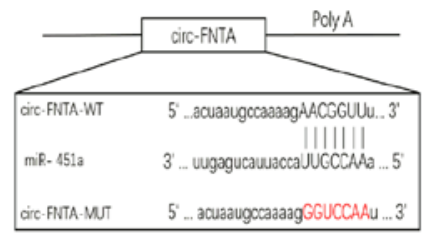

C

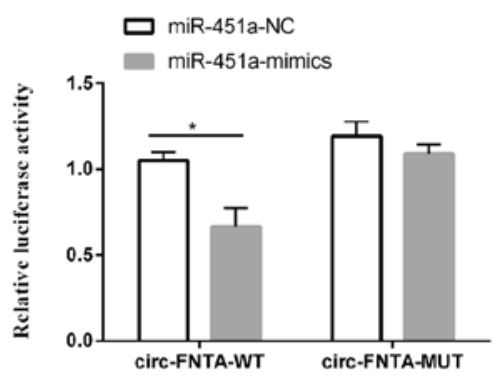

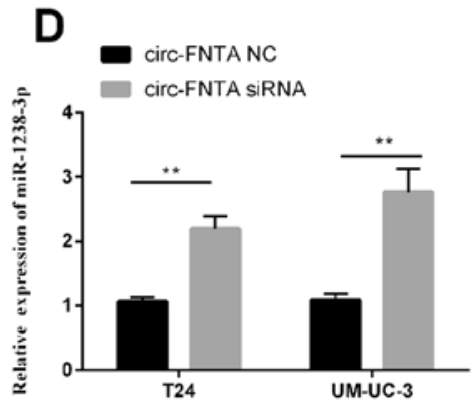

$\mathbf{E}$

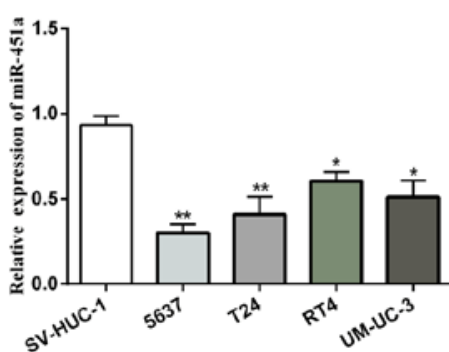

Figure 3. circ-FNTA targets miR-451a. (A) Potential binding miRNAs to circ-FNTA predicted by Starbase and Targetscan. (B) Potential binding sites between circ-FNTA and miR-451a. (C) Luciferase activity in T24 cells co-transfected with pmirGLO-circ-FNTA-mut/pmirGLO-circ-FNTA-wt and miR-415a mimics/NC (compared with NC, ${ }^{*} \mathrm{P}<0.05$ ). (D) Relative level of miR-451a in UM-UC-3 and T24 cells transfected with si-NC or si-circ-FNTA \#1 (compared with si-NC, $\left.{ }^{* *} \mathrm{P}<0.01\right)$. (E) Relative level of miR-451a in human bladder immortalized epithelial cells (SV-HUC-1) and BCa cells (5637, T24, RT4 and UM-UC-3) (compared with SV-HUC-1, $\left.{ }^{*} \mathrm{P}<0.05,{ }^{* *} \mathrm{P}<0.01\right)$. BCa, bladder cancer.

A

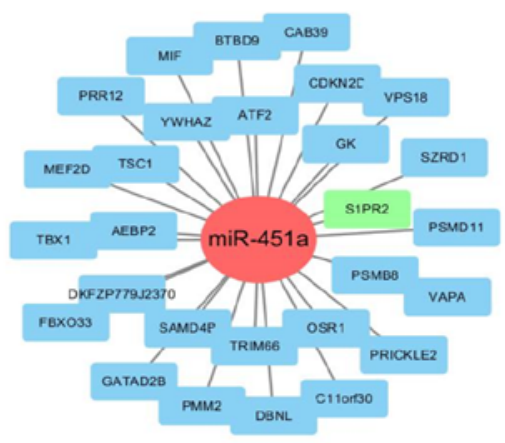

C

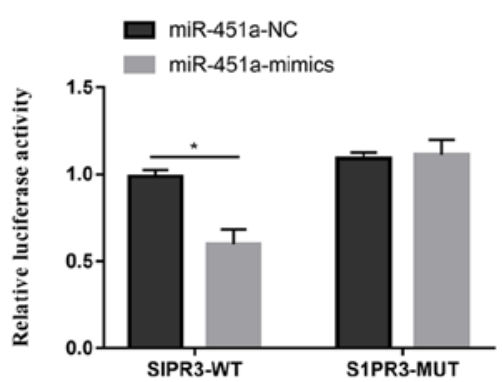

B

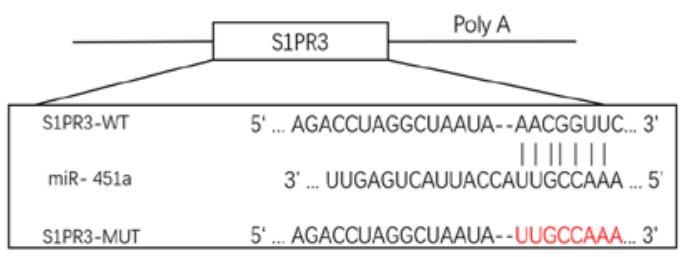

D

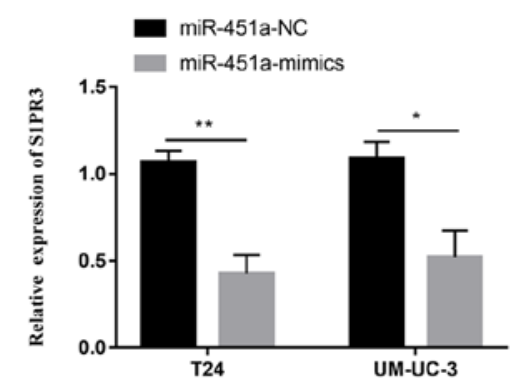

E

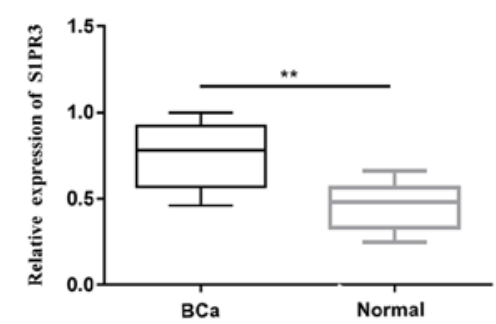

Figure 4. miR-451a regulates its target gene S1PR3. (A) Potential binding targets of miR-451a. (B) Potential binding sites between miR-451a and S1PR3. (C) Luciferase activity in T24 cells co-transfected with pmirGLO-S1PR3-mut/pmirGLO-S1PR3-wt and miR-415a mimics/NC (compared with NC, ${ }^{*} \mathrm{P}<0.05$ ). (D) Relative expression of S1PR3 in UM-UC-3 and T24 cells transfected with NC or miR-451a mimics (compared with NC, $\left.{ }^{*} \mathrm{P}<0.05,{ }^{* *} \mathrm{P}<0.01\right)$. (E) Relative level of S1PR3 in BCa tissues and normal tissues (compared with normal, $\left.{ }^{* * *} \mathrm{P}<0.01\right)$. BCa, bladder cancer. 
CeRNA theory proposes that circRNA sponges miRNA to influence the target gene expression, thus influencing the pathological progression (28).

S1PR3 (sphingosine-1 phosphate receptor 3) is a key receptor gene for tumor progression. For example, in lung adenocarcinoma, S1PR3 expression is upregulated and closely related to the activated TGF- $\beta /$ SMAD pathway. S1PR3 activation can promote malignant progression of lung cancer (29). In addition, S1PR3 induces expansion of cancer stem cells by activating Notch signaling pathway, and S1PR3 may be a potential target for tumor therapy (30). S1PR3 is a molecular marker for tumor progression of $\mathrm{BCa}$, which exerts prognostic potential (21). It is suggested that S1PR3 has a carcinogenic role in aggravating the malignant progression of tumors.

In this study, circ-FNTA was upregulated in BCa tissues and cell lines. Through analyzing the clinical data of BCa patients, circ-FNTA was found to be highly expressed in invasive $\mathrm{BCa}$ patients relative to the non-invasive ones. In vitro experiments demonstrated that silence of circ-FNTA attenuated proliferative and invasive abilities of BCa. Subsequently, through online prediction and dual-luciferase reporter gene assay verification, miRNA-451a was confirmed to be the target of circ-FNTA and S1PR3 was found to be the target gene of miRNA-451a. Our study identified the role of circ-FNTA/miRNA-451a/S1PR3 axis in aggravating the progression of $\mathrm{BCa}$.

In conclusion, circ-FNTA accelerates the proliferative and invasive abilities of BCa through absorbing miRNA-451a to regulate the S1PR3 level, and indicates a poor prognosis of BCa patients.

\section{Acknowledgements}

Not applicable.

\section{Funding}

No funding was received.

\section{Availability of data and materials}

All data generated or analyzed during this study are included in this published article.

\section{Authors' contributions}

JT and LZ designed the study and performed the experiments, JT and JF established the animal models, LZ and JX collected the data, TR and HG analyzed the data, JT and LZ prepared the manuscript. All the authors read and approved the final manuscript.

\section{Ethics approval and consent to participate}

This study was approved by the Ethics Committee of Linyi Cancer Hospital (Linyi, China). Signed informed consents were obtained from the patients and/or guardians.

\section{Patient consent for publication}

Not applicable.

\section{Competing interests}

The authors declared no conflict of interest.

\section{References}

1. Siegel RL, Miller KD, Fedewa SA, Ahnen DJ, Meester RGS, Barzi A and Jemal A: Colorectal cancer statistics, 2017. CA Cancer J Clin 67: 177-193, 2017.

2. Soerjomataram I, Lortet-Tieulent J, Parkin DM, Ferlay J, Mathers C, Forman D and Bray F: Global burden of cancer in 2008: A systematic analysis of disability-adjusted life-years in 12 world regions. Lancet 380: 1840-1850, 2012.

3. Chen D, Li SG, Chen JY and Xiao M: miR-183 maintains canonical Wnt signaling activity and regulates growth and apoptosis in bladder cancer via targeting AXIN2. Eur Rev Med Pharmacol Sci 22: 4828-4836, 2018.

4. Stone L: Bladder cancer: Mastering the immune microenvironment. Nat Rev Urol 14: 639, 2017.

5. Sargos P, Baumann BC, Eapen L, Christodouleas J, Bahl A, Murthy V, Efstathiou J, Fonteyne V, Ballas L, Zaghloul M, et al: Risk factors for loco-regional recurrence after radical cystectomy of muscle-invasive bladder cancer: A systematic-review and framework for adjuvant radiotherapy. Cancer Treat Rev 70: 88-97, 2018.

6. Grossman HB: Bladder cancer: Neoadjuvant is new again. Lancet Oncol 12: 830-831, 2011.

7. Morales-Barrera R, Suárez C, de Castro AM, Racca F, Valverde C, Maldonado X, Bastaros JM, Morote J and Carles J: Targeting fibroblast growth factor receptors and immune checkpoint inhibitors for the treatment of advanced bladder cancer: New direction and New Hope. Cancer Treat Rev 50: 208-216, 2016.

8. Liu X, Abraham JM, Cheng Y, Wang Z, Wang Z, Zhang G, Ashktorab H, Smoot DT, Cole RN, Boronina TN, et al: Synthetic circular RNA functions as a miR-21 sponge to suppress gastric carcinoma cell proliferation. Mol Ther Nucleic Acids 13: 312-321, 2018.

9. Hansen TB, Kjems J and Damgaard CK: Circular RNA and miR-7 in cancer. Cancer Res 73: 5609-5612, 2013.

10. Liu J, Liu T, Wang X and He A: Circles reshaping the RNA world: From waste to treasure. Mol Cancer 16: 58, 2017.

11. Lasda E and Parker R: Circular RNAs: Diversity of form and function. RNA 20: 1829-1842, 2014.

12. Liu Y, Feng J, Sun M, Yang G, Yuan H, Wang Y, Bu Y, Zhao M, Zhang $S$ and Zhang X: Long non-coding RNA HULC activates HBV by modulating HBx/STAT3/miR-539/APOBEC3B signaling in HBV-related hepatocellular carcinoma. Cancer Lett 454: 158-170, 2019.

13. Zhang B, Chen M, Jiang N, Shi K and Qian R: A regulatory circuit of circ-MTO1/miR-17/QKI-5 inhibits the proliferation of lung adenocarcinoma. Cancer Biol Ther 20: 1127-1135, 2019.

14. He J, Chen J, Ma B, Jiang L and Zhao G: CircLMTK2 acts as a novel tumor suppressor in gastric cancer. Biosci Rep 39: 39, 2019.

15. Zhong Z, Lv M and Chen J: Screening differential circular RNA expression profiles reveals the regulatory role of circTCF25-miR-103a-3p/miR-107-CDK6 pathway in bladder carcinoma. Sci Rep 6: 30919, 2016.

16. Glažar P, Papavasileiou P and Rajewsky N: circBase: A database for circular RNAs. RNA 20: 1666-1670, 2014.

17. Sebti SM and Adjei AA: Farnesyltransferase inhibitors. Semin Oncol 31 (Suppl 1): 28-39, 2004.

18. Li JH, Liu S, Zhou H, Qu LH and Yang JH: starBase v2.0: Decoding miRNA-ceRNA, miRNA-ncRNA and protein-RNA interaction networks from large-scale CLIP-Seq data. Nucleic Acids Res 42D: D92-D97, 2014.

19. Agarwal V, Bell GW, Nam JW and Bartel DP: Predicting effective microRNA target sites in mammalian mRNAs. eLife 4: $4,2015$.

20. Matsushita R, Seki N, Chiyomaru T, Inoguchi S, Ishihara T, Goto Y, Nishikawa R, Mataki H, Tatarano S, Itesako T, et al: Tumour-suppressive microRNA-144-5p directly targets CCNE1/2 as potential prognostic markers in bladder cancer. Br J Cancer 113: 282-289, 2015.

21. Go H, Kim PJ, Jeon YK, Cho YM, Kim K, Park BH and Ku JY: Sphingosine-1-phosphate receptor 1 (S1PR1) expression in non-muscle invasive urothelial carcinoma: Association with poor clinical outcome and potential therapeutic target. Eur J Cancer 51: 1937-1945, 2015. 
22. Feng J, Chen K, Dong X, Xu X, Jin Y, Zhang X, Chen W, Han Y, Shao L, Gao Y, et al: Genome-wide identification of cancer-specific alternative splicing in circRNA. Mol Cancer 18: 35, 2019.

23. Su H, Tao T, Yang Z, Kang X, Zhang X, Kang D, Wu S and Li C: Circular RNA cTFRC acts as the sponge of microRNA-107 to promote bladder carcinoma progression. Mol Cancer 18: 27, 2019.

24. Gu C, Zhou N, Wang Z, Li G, Kou Y, Yu S, Feng Y, Chen L, Yang $\mathrm{J}$ and Tian F: circGprc5a promoted bladder oncogenesis and metastasis through Gprc5a-targeting Peptide. Mol Ther Nucleic Acids 13: 633-641, 2018.

25. Chen X, Chen RX, Wei WS, Li YH, Feng ZH, Tan L, Chen JW, Yuan GJ, Chen SL, Guo SJ, et al: PRMT5 circular RNA promotes metastasis of urothelial carcinoma of the bladder through sponging miR-30c to induce epithelial-mesenchymal transition. Clin Cancer Res 24: 6319-6330, 2018.

26. Dangle PP, Zaharieva B, Jia H and Pohar KS: Ras-MAPK pathway as a therapeutic target in cancer - emphasis on bladder cancer. Recent Patents Anticancer Drug Discov 4: 125-136, 2009.

27. Chin K, DeVries S, Fridlyand J, Spellman PT, Roydasgupta R, Kuo WL, Lapuk A, Neve RM, Qian Z, Ryder T, et al: Genomic and transcriptional aberrations linked to breast cancer pathophysiologies. Cancer Cell 10: 529-541, 2006.
28. Qi X, Lin Y, Chen J and Shen B: Decoding competing endogenous RNA networks for cancer biomarker discovery. Brief Bioinform: Jan 30, 2019 (Epub ahead of print). doi.org/10.1093/bib/bbz006.

29. Zhao J, Liu J, Lee JF, Zhang W, Kandouz M, VanHecke GC, Chen S, Ahn YH, Lonardo F and Lee MJ: TGF- $\beta$ /SMAD3 pathway stimulates sphingosine-1 phosphate receptor 3 expression: Implication of sphingosine-1 phosphate receptor 3 in lung adenocarcinoma progression. J Biol Chem 291: 27343-27353, 2016.

30. Hirata N, Yamada S, Shoda T, Kurihara M, Sekino Y and Kanda Y: Sphingosine-1-phosphate promotes expansion of cancer stem cells via S1PR3 by a ligand-independent Notch activation. Nat Commun 5: 4806, 2014.

This work is licensed under a Creative Commons Attribution-NonCommercial-NoDerivatives 4.0 International (CC BY-NC-ND 4.0) License. 